\title{
Gestion des écosystèmes pâturés en situations extensives : apports de l'écologie fonctionnelle et perspectives de recherches appliquées en moyenne montagne humide
}

\author{
Pierre Loiseau ${ }^{\mathrm{a} *}$, Frédérique Louault ${ }^{\mathrm{a}}$, Gérard L'Homme ${ }^{\mathrm{b}}$ \\ "Inra, unité d"agronomie, fonctionnement et gestion de l'écosystème prairial, Domaine de Crouël, \\ 234, avenue du Brézet, 63039 Clermont-Ferrand cedex 2, France \\ ${ }^{\mathrm{b}}$ Enita Clermont-Ferrand, Marmilhat, Lempdes, France
}

(Reçu le 24 avril 1998 : accepté le 24 août 1998)

\begin{abstract}
Management of grazed ecosystems in extensified conditions: Relevance of functional ecology and research prospects, applied to moist middle mountains. According to the situations encountered in extensified breeding farms, three important pieces of ecological knowledge allow to understand grassland ecosystem dynamics, in order to settle sustainable pasture management in both production and environmental aims. i) The interaction between vegetation and herbivores must be considered as well as the long term adaptation between vegetation status and stocking rate, or as the instantaneous modulation of grassland production and structure under herbivore predation. ii) The dynamics of botanical composition must be represented in terms of sensitivity and resilience to perturbations or to management changes, which condition primary phytomass production and accumulation. Extensifying grassland management induces reversible effects on the seasonal dynamics of the phytomass, but cumulative negative effects on botanical composition. Species strategies are involved in the dynamics of the spatial structure and diversity of the vegetation. iii) The management of the nitrogen eycle allows to manipulate in a long term the soil nitrogen supplying capacity, for improving the perennity of sowed swards, or making easier the control of the phytomass under low stocking rates. The implications for $\mathrm{N}$ leaching are discussed. (1) Elsevier / Inra
\end{abstract}

dynamics / management / grassland / grazing / biogeochemical cycle

Résumé - Compte tenu des situations rencontrées dans les fermes d'élevage extensives, trois facteurs écologiques expliquent la dynamique des écosystèmes prairiaux et s'appliquent à la mise au point des systèmes de pâturage conciliant les objectifs de production et d'environnement. i) Les interactions

\footnotetext{
* Correspondance et tirés à part

Tél. : (33) 0473624427 ; fax : (33) 0473624457 ; e-mail : loiseau@ valmont.clermont.inra.fr
} 
entre les états de végétation et les effets des herbivores doivent s'envisager depuis les lois d'équilibres à long terme entre le chargement et la composition botanique jusqu'aux modifications instantanées de la production et de la structure par la prédation. ii) La dynamique de la composition botanique doit être caractérisée par la sensibilité et la résilience des prairies aux perturbations ou aux changements de la gestion qui conditionnent notamment la production primaire et l'accumulation des phytomasses. L'extensification de la gestion induit des changements instantanés et réversibles des dynamiques saisonnières de phytomasses, mais des effets cumulatifs défavorables sur la composition botanique. Les stratégies d'espèces doivent permettre de rendre compte de l'évolution de la structuration spatiale et de la diversité des communautés. iii) La gestion du cycle de l'azote permet de manipuler à long terme le pouvoir de fourniture des sols en azote, soit pour améliorer la pérennité des prairies semées, soit pour faciliter le contrôle des phytomasses en conditions de cheptel limitant. Les implications pour le lessivage des nitrates sont discutées. (Elsevier / Inra

\section{dynamique / gestion / prairie / pâturage / cycle biogéochimique}

\section{INTRODUCTION}

L'intensification des systèmes d'élevage et de pâturage a permis une augmentation très importante de la productivité herbagère et animale au prix d'effets négatifs sur l'environnement. L'enjeu pour le futur, en développant des systèmes d'élevage plus extensifs, est de gérer l'ensemble de l'espace fourrager, en respectant à la fois les objectifs et les contraintes de production et d'environnement. Pour les systèmes intensifs, il s'agit de limiter les effets négatifs sur l'environnement. Pour les systèmes moins intensifs, le problème consiste à concilier production et entretien de l'espace, en préservant la diversité végétale et la propreté des eaux de drainage.

Les objectifs de cette communication sont de présenter un état des connaissances écologiques et agronomiques en matière de fonctionnement de l'écosystème pâturé et de dégager les perspectives de recherche appliquée qui contribueront à la mise au point de nouveaux modes de gestion des espaces fourragers à l'échelle des unités de gestion (lots d'animaux et parcelles). Les contextes pris comme référence sont les prairies pérennes des régions d'élevage de moyenne montagne. Compte tenu de la diversité des milieux et des fonctions des parcelles dans les exploitations extensives, nous développerons quelques aspects essentiels des mécanismes qui déterminent la valeur d'usage et la dynamique des prairies selon leur gestion : i) les relations entre végétation et herbivore, ii) l'évolution des communautés végétales en condition de sous-exploitation et iii) les flux et bilans d'azote dans les écosystèmes sol-peuplement prairial.

\section{2. ÉQUILIBRE ENTRE LA VÉGÉTATION ET L'HERBIVORE}

Une production durable des systèmes de pâturage exige des quantités et qualités ingérées suffisantes par animal pendant le séjour d'un groupe sur une parcelle. Cette exigence s'applique à l'ensemble des unités parcellaires dont les fonctions complémentaires sont organisées pour réguler le système fourrager $[4,5,19,26]$. Plusieurs critères dirigent les choix de gestion au sein de l'exploitation : la diversité écologique - naturelle ou acquise - des différentes parcelles [9], les facilités d'exploitation liées à la structure foncière [28] et l'hétérogénéité interne des parcelles, naturelle ou due à un regroupement foncier [8]. Une typologie basée sur les fonctions agricoles et les caractéristiques des parcelles distingue deux situations et problématiques de gestion : 
1) Des parcelles riches, mécanisables et homogènes, qui fournissent l'essentiel des ressources pâturées par les lots d'animaux les plus exigeants et permettent de reconstituer les stocks. Une production de bonne qualité est obtenue par un taux élevé d'utilisation de la production. Un objectif essentiel est d'optimiser les entrées d'azote par les engrais et par la fixation symbiotique des légumineuses pour obtenir un meilleur rapport entre les coûts et les bénéfices en limitant les impacts environnementaux et en pérennisant la capacités de production du milieu.

2) Des parcelles de moindres aptitudes agronomiques, plus pauvres, éloignées ou hétérogènes, qui sont pâturées par des lots d'animaux moins exigeants ou en complément par les lots principaux. Le taux de consommation de la production est faible et variable dans l'espace et le temps. Les problèmes de maîtrise des aptitudes pastorales se posent pour de telles parcelles, qu'elles soient situées dans les milieux pauvres ou, dans les milieux riches, gérés jusqu'alors de manière plus intensive [4].

En systèmes allaitants, les objectifs de production sont atteints avec une faible artificialisation du milieu. Les interventions directes de l'éleveur sur le sol se réduisent souvent à la gestion des fertilisants. Une proportion importante des surfaces fourragères est gérée essentiellement par herbivore interposé. Dans ces conditions, le fonctionnement et l'évolution de l'écosystème prairial reposent sur des interactions entre les producteurs, les consommateurs et les décomposeurs de matières organiques. Gérer un écosystème pâturé, revient à piloter ces interactions pour réaliser les objectifs que sont la production animale et le maintien du milieu qui permet de la perpétuer. Les nouveaux objectifs d'agriculture durable imposent une meilleure connaissance des régulations internes responsables des propriétés adaptatives et évolutives des écosystèmes complexes.

\subsection{Relation entre la composition botanique et le chargement dans un milieu donné}

Le chargement est le critère le plus global pour estimer l'impact du troupeau sur la végétation et le milieu dans des situations à l'équilibre. Dans les montagnes d'estive extensives du Massif Central, Daget et Poissonnet [10] ont montré que le chargement animal était corrélé positivement à l'abondance des bonnes espèces fourragères. Cette relation a permis d'élaborer un diagnostic de la valeur d'usage des communautés végétales par un indice de valeur pastorale (VP) basé sur la composition botanique et sur une expertise des espèces composantes des communautés. L'indice de VP a été largement utilisé dans les opérations de recherchedéveloppement en estives [29, 42]. L'analyse factorielle de la composition botanique des communautés a fourni un champ d'hypothèses sur les relations à long terme entre la gestion et l'état des prairies [3, 12], dont la valeur prédictive a été démontrée par des études diachroniques en situation réelle chez les éleveurs [17, 31].

L'interprétation fonctionnelle de la corrélation entre VP et chargement implique une plasticité de la végétation et introduit le concept de pouvoir améliorateur de l'herbivore sur sa pâture. Une approche expérimentale a été réalisée en Auvergne dans les années 1970 à 1980 [43] en imposant des chargements croissants à une même végétation initiale. Les expériences ont démontré que le chargement n'était pas seulement une conséquence mais un déterminant de la VP. Pour un milieu et une conduite du pâturage donnés, il existe un chargement $\mathrm{Chm}$ qui maximise les aptitudes fourragères de la végétation. Dans des milieux initialement pauvres, l'augmentation de la VP avec le chargement jusqu'à Chm s'explique par un effet positif de l'herbivore sur la disponibilité en éléments nutritifs pour la croissance de l'herbe [45]. La diminution de la VP au delà de Chm s'explique par un effet négatif 
de la défoliaison sélective des meilleures espèces renforçant la compétitivité des indésirables $[1,23,30]$.

\subsection{Application à la répartition d'un cheptel sur un territoire}

À l'étage collinéen, une relation similaire entre la VP et le chargement a été proposée [34] (figure l). Elle permet de raisonner globalement la répartition sur le territoire d'un cheptel limité, considéré comme outil d'entretien de la végétation herbacée. À l'équilibre, Chm maximise la valeur des surfaces entretenues mais minimise leur étendue, laissant une grande partie du territoire à la forêt. Le plus fort rapport entre la qualité de la végétation à l'équilibre et le chargement est obtenu pour une charge critique d'intervention (Chc) qui maximise la ressource fourragère sur l'ensemble du territoire. Au chargement d'entretien de l'herbe (Che), la proportion de surface entretenue est maximisée, mais la surface en forêt est minimisée au prix d'une faible valeur fourragère de la surface en herbe et de difficultés pour y contrôler le développement des espèces non fourragères.
De plus, les territoires à entretenir présentent une grande diversité selon les parcelles. Les niveaux de chargement critiques sont plus élevés dans les milieux riches que dans les milieux pauvres. En conséquence, à un même chargement faible, les surfaces préalablement intensifiées de la SAU poseront des problèmes de maîtrise plus aigus que celle des anciens parcours situés sur les milieux pauvres. Au sein d'une parcelle hétérogène soumise à une diminution du chargement, la dégradation de la végétation risque d'être plus rapide dans les zones riches ou dans les zones pauvres selon la gestion du pâturage. Pour apprécier cette dynamique, il est nécessaire de mieux connaître les lois de comportement des herbivores en fonction de la répartition des ressources à l'intéricur des parcelles [42].

\subsection{Interaction entre production et exploitation}

Dans un même milieu, la production instantanée sous pâturage varie dans une large mesure selon la gestion [15]. Les progrès en écophysiologie des peuplements (Soussana et al., présent ouvrage) permettent la

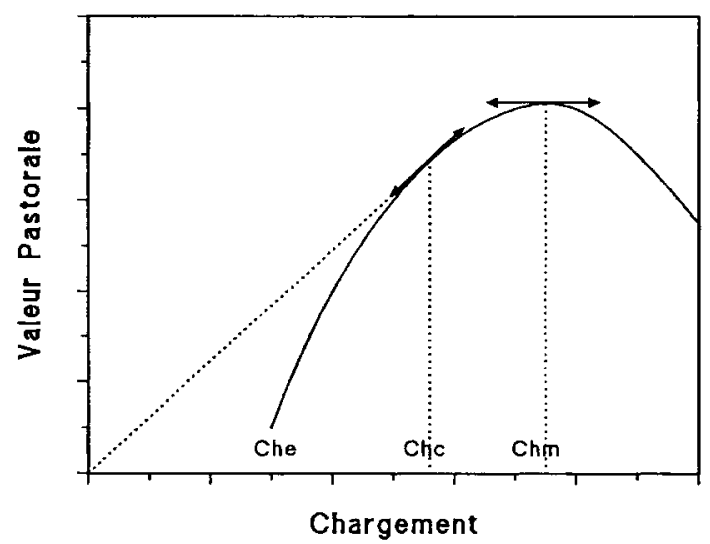

Figure 1. Relation à l'équilibre entre le chargement en bétail et la composition botanique représentéc par l'indice de valeur pastorale (VP). Che : charge qui maximise la surface entretenue par le troupeau ; Che : charge critique qui maximise la ressource fourragère ; Chm : charge qui maximise la valeur de la partie entretenue. 
mesure et la modélisation de la croissance réelle des couverts défoliés selon les ressources en nutriments [40]. De nouvelles possibilités sont ainsi offertes pour comprendre et piloter les états de l'herbe pâturée [14]. Dans les expérimentations de pâturage à hauteur constante, un chargement identique en animaux peut induire différentes hauteurs et productions d'herbe $[6,36,38]$. Aux faibles hauteurs d'herbe, la production est limitée par l'indice foliaire et le taux de consommation de la production réelle est important. À des hauteurs d'herbe élevées, la production est plus importante mais un faible taux de consommation de la production réduit la qualité du fourrage offert [40]. La production primaire et son taux de consommation pour un milieu et un chargement donnés dépendent en partie de la gestion antérieure $[40,44]$, soulignant la nécessité d'approches dynamiques. L'herbe est plus haute si le chargement est atteint à partir de pressions d'utilisation antérieures plus faibles. Anticiper ces situations permet en particulier aux éleveurs de raisonner les techniques complémentaires d'intervention mécanique capables de maîtriser une trop forte accumulation de biomasse sur les pâturages extensifiés [9].

\section{DYNAMIQUE \\ DE L'ÉCOSYSTÈME PÂTURÉ}

\subsection{Caractérisation sur l'exemple du facteur trophique}

Les perturbations qui affectent la réorganisation des écosystèmes consistent soit en phénomènes temporaires caractérisés par leur fréquence et leur intensité [25, 51], soit en modifications durables d'un facteur écologique [21]. Le délai nécessaire pour le retour d'une variable d'état à l'état d'équilibre initial après une perturbation instantanée définit l'élasticité de cette variable face à la perturbation [24]. La variable est durablement modifiée dès que la durée moyenne entre deux perturbations excède l'élasticité.
Ainsi, après une perturbation déclenchée par une concentration locale du piétinement et des déjections du troupeau, l'élasticité de la production standard et de la composition botanique de parcours pauvres est supérieure à 5 ans. Dans la pratique, les éleveurs de la chaîne des Dômes appliquent ces perturbations tous les 3 à 5 ans, dans un but d'amélioration du parcours [33].

Il est important de pouvoir caractériser l'inertie de la végétation ou de formuler des diagnostics précoces de la tendance évolutive, afin de maintenir la réversibilité par une adaptation de la gestion. Dans le cas de pratiques modifiant le facteur trophique, la relation entre les variables de production et de composition botanique de peuplements à l'équilibre permet une interprétation des dynamiques d'évolution [31]. Un apport fertilisant ou un épuisement du sol par l'exportation provoquent une évolution plus rapide de la production que de la composition botanique $[33,34]$. La durée nécessaire à l'obtention d'un rapport conforme à l'équilibre traduit l'inertie ou résilience de l'écosystème (5 ans pour les pelouses à Nardus stricta $[25,39])$. Le rapport entre ces deux variables comparé à leur rapport à l'équilibre traduit le sens de la dynamique instantanée d'un écosystème face au facteur considéré.

\subsection{Application à la sous-exploitation des milieux riches}

Une perturbation importante intervenant sur un système à faible inertie peut provoquer la disparition d'espèces fourragères [38]. En conditions de moyenne montagne en Auvergne, la composition botanique d'une prairie extensifiée se dégrade par disparition des bonnes espèces fourragères (Trifolium repens, Lolium perenne) et augmentation d'espèces moins bonnes (Elymus sp.). À l'équilibre, le niveau de dégradation augmente avec le degré d'extensification (figure 2a). La composition botanique d'une prairie conduite alternativement de façon extensifiée et selon un itinéraire technique 

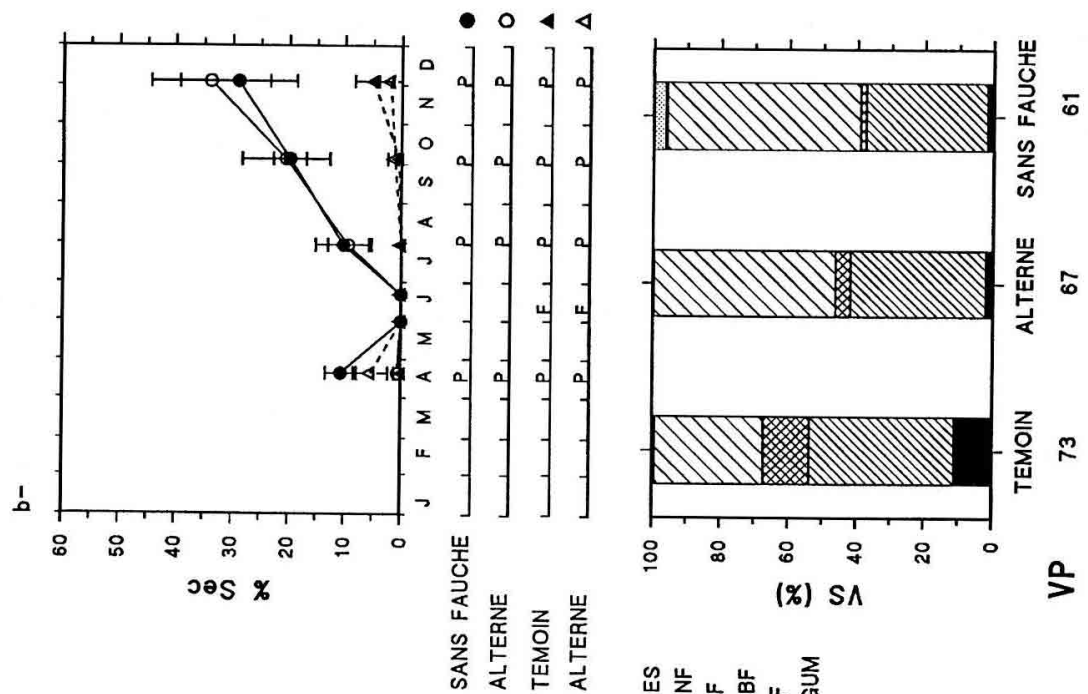

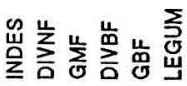
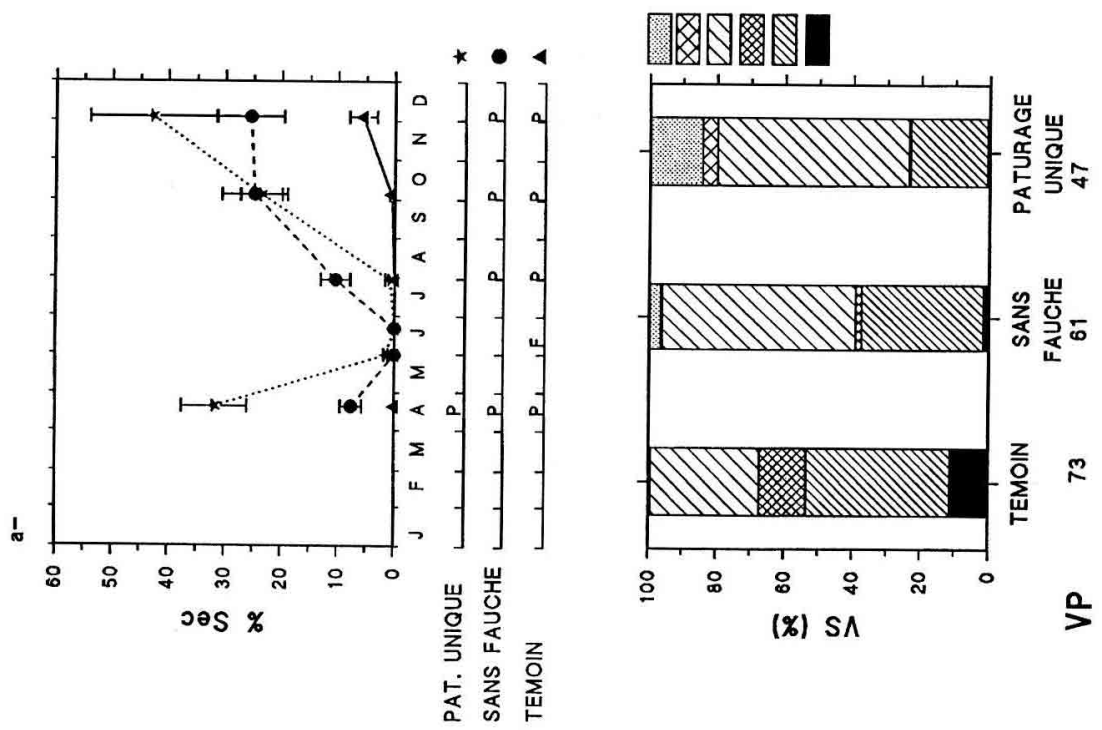

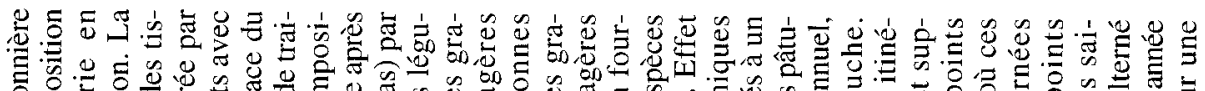

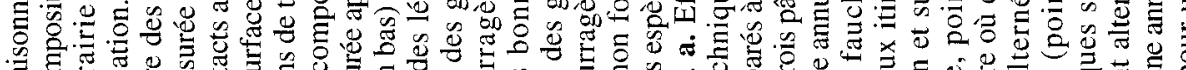

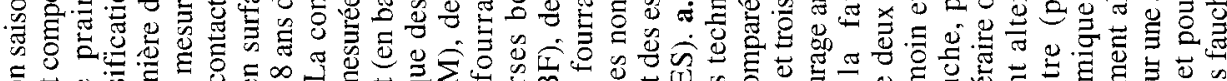

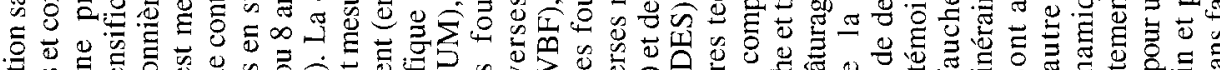

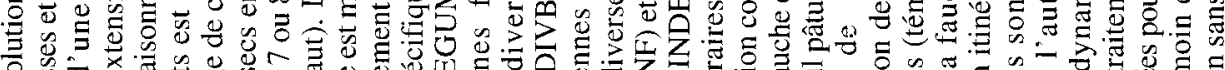

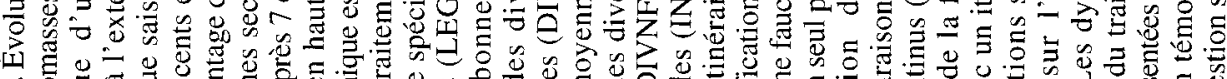

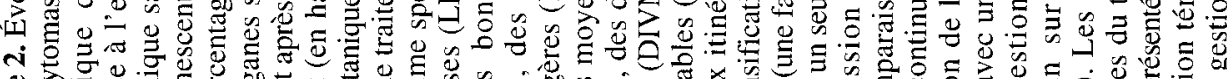

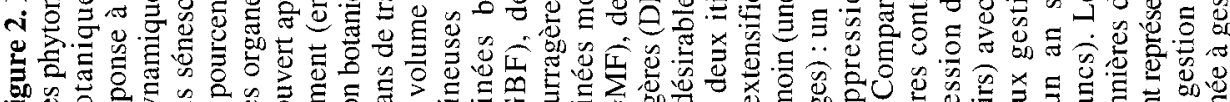

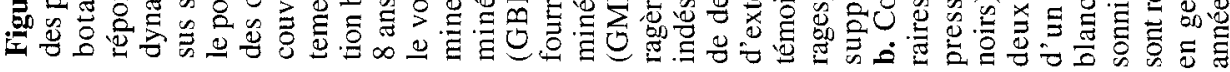


d'entretien prend des valeurs intermédiaires entre celles obtenues par les mêmes conduites pratiquées de façon continue (figure $2 b$ ). La composition botanique est donc sensible aussi bien au degré d'extensification qu'au nombre cumulé d'années de conduite extensifiée.

La sensibilité de la végétation aux perturbations résulte de régulations internes, importantes à élucider, qui conditionnent la souplesse d'adaptation des prairies à la gestion. L'identification des fonctions adaptatives des espèces responsables de leur compétitivité dans les communautés apparaît nécessaire pour une compréhension de l'évolution, applicable à différents états initiaux de communautés. En conséquence, l'évolution botanique devra être considérée non plus en termes d'espèces, mais de groupes d'espèces-type, distinguées par les caractéristiques biologiques qui déterminent leur comportement face au facteur actif : physiologie, morphologie, cycle saisonnier. Si les stratégies trophiques permettent de comprendre la dynamique des végétations soumises à des variations de la disponibilité en minéraux, les stratégies clonales qui déterminent l'architecture, l'intégration physiologique et le cycle annuel des espèces [49] conditionnent fortement leur adaptation à l'intensité d'exploitation. La compétition pour la lumière [37] favorise le développement d'espèces à forte plasticité phénotypique. Les stratégies d'expansion par rhizomes ou stolons sont favorisées avec Ranunculus repens, Poa pratensis, Agrostis tenuis [37, 50] et Elymus sp. (figure 2). Alors que la pérennité végétative est souvent prédominante dans les prairies bien gérées, la sous-exploitation renforce le rôle de la reproduction par graine dans la dynamique de végétation [36b]. En conséquence, les reconversions d'usage peuvent se traduire par l'introduction d'espèces nouvelles, dépendant de l'environnement de l'écosystème et susceptibles d'augmenter la diversité écologique des espèces au sein de la communauté [4].
La sous-exploitation modifie aussi l'importance et la composition des phytomasses qui conditionnent leur valeur d'usage pour l'herbivore. La dynamique saisonnière des stocks sur pied dépend essentiellement de l'itinéraire technique de l'année en cours et ne montre pas d'effets cumulés du nombre d'années de traitement (figure 2b). La fauche et le pâturage d'arrière saison apparaissent donc comme des moyens puissants de recréer des états d'herbe favorables à une augmentation de la pression de pâturage et à une restauration à terme de la qualité botanique des prairies dégradées par un souspâturage $[9,50]$.

Pratiquement, l'intensité d'exploitation se traduit donc de façon immédiate sur la structure du peuplement, et à plus long terme sur l'évolution botanique. Les recherches futures devront caractériser l'élasticité de l'écosystème par rapport à ces deux variables pour préciser les conditions de réversibilité de la dynamique par la gestion. L'expertise classique de la valeur d'usage de la prairie par les indices spécifiques devra alors être remplacée par des concepts de stratégie d'espèce.

\subsection{Rôle de l'hétérogénéité dans la dynamique des formations herbacées}

Dans les parcelles exploitées en pâturage extensif, les stratégies de croissance et de morphogenèse des espèces végétales participent à l'hétérogénéisation de la structure verticale et horizontale du couvert. Le renforcement du pouvoir compétitif des espèces à capacité de déplacement horizontal et des espèces capables de ports cespiteux se traduit par l'arrangement spatiotemporel des communautés en plusieurs faciès $[22,27$, $51,52]$, dominés chacun par un nombre limité de stratégies adaptatives. Cette évolution des communautés associe donc une diminution de la diversité spécifique interne aux faciès ( $\alpha$-diversité) à une augmentation de la diversité en faciès ( $\beta$-diversité). Les conséquences de l'hétérogénéisation pour 
la physionomie des prairies trouvent des applications en matière de diagnostic agronomique $[18,20]$. Les recherches futures auront à mieux comprendre cette dynamique de patchs, en particulier à préciser dans quelle mesure l'hétérogénéité du milieu [38c] participe à la structuration spatiale de la végétation [49] ou en résulte.

Dans les pâturages extensifs soumis à un chargement constant, de nouvelles hypothèses fondées sur les interactions entre herbe et herbivore doivent fonder de futures recherches sur l'apparition, la nature, le rôle et la gestion de l'hétérogénéité dans les écosystèmes pâturés [7]. Les hauteurs d'herbe et les rapports de compétition entre faciès, induits par des facteurs internes à la végétation, sont modulables par des facteurs externes tels que les préférences alimentaires des animaux $[1,23]$ ou la sensibilité au piétinement. De telles recherches deviennent nécessaires pour une application aux couverts extensifiés-sénescents.

\subsection{Rôle de l'hétérogénéité dans la dynamique des formations mixtes herbacées-ligneux}

À des niveaux de chargement voisins de la charge d'entretien Che, les communautés évoluent en complexes de synusies d'herbe et de ligneux temporellement et spatialement dépendantes [22]. Les processus en cause dans la maintenance de ces formations sont à la fois de natures interne et externe. Les premiers concernent la capacité d'accumulation, la vitesse de vieillissement puis de régénération des ligneux et mettent en cause le cycle des éléments [52]. Les seconds interagissent par la plus ou moins grande sensibilité des végétations aux actions physiques du climat (gel sur Sarothamnus scoparius [46]), de l'homme (feux) et du troupeau (rajeunissement des landes à Calluna vulgaris [32]). Faute d'une maintenance par la gestion de cette dynamique cyclique, une évolution se déclenche vers le boisement et la fermeture de l'espace. En conséquence, au voisinage de la charge d'entretien, la gestion de ces végétations doit viser le maintien de la dispersion spatiale et temporelle des patchs. Le débroussaillage mécanique instantané de surfaces trop importantes est à prohiber car une augmentation trop rapide de la surface à pâturer implique à moyen terme une augmentation de la surface en friche et des risques d'évolution vers la forêt.

\section{CYCLE DE L'AZOTE DANS L'ÉCOSYSTÈME PÂTURÉ}

La disponibilité en azote minéral conditionne i) la production de biomasse, ii) l'évolution de la composition botanique, iii) les risques de pollution nitrique des eaux de ruissellement et de drainage. Dans les systèmes intensifiés, le niveau d'alimentation en azote est souvent caractérisé par celui de la fertilisation minérale. En système extensif, l'alimentation en azote dépend essentiellement de la disponibilité des éléments biogènes des sols. Une connaissance du cycle de l'azote et de ses liaisons avec le cycle du carbone dans le système sol-peuplement devient alors nécessaire pour mieux contrôler les états de l'écosystème. Nous examinerons seulement quelques applications de la gestion du bilan de l'azote au contrôle du pouvoir de fourniture en azote des sols et de la pollution nitrique des eaux de drainage.

\subsection{Bilan environnemental de l'azote et contrôle de la fertilité des sols}

Le bilan des flux d'entrée et de sortie d'azote les plus facilement mesurables du système pâturé (apports fertilisants et exportations dans les produits animaux et végétaux) permet d'apprécier l'évolution des aptitudes du milieu [11, 16, 47], mais, selon les situations, il se répercute plus ou moins sur les pertes d'azote ou sur le bilan azoté du sol. Dans les systèmes prairiaux fauchés, 
pauvres en légumineuses et sujets à de faibles pertes [13], une diminution du bilan de l'azote réduit le pouvoir de foumiture en azote du sol et restaure la diversité des prairies intensifiées [41]. Dans les systèmes pâturés, un bilan négatif entraîne l'appauvrissement des terrains de parcours, dont la capacité de production épigée est réduite de 4,8 à 2,8 tMS/ha/an en trente ans [31 ]. Dans des prairies sans légumineuses, un régime de fauche sans restitutions diminue de moitié l'azote mobilisé dans les récoltes en 3 à 7 ans selon l'état initial de la prairie (tableau I).

Inversement, les bilans positifs augmentent à terme le bilan du sol, sa capacité de fourniture en azote à long terme et la capacité ultérieure de production de la prairie. Ainsi, au bout de 4 ans, la production à l'apport nul d'une prairie fauchée (Festuca arundinacea) recevant depuis le semis soit 150 , soit $350 \mathrm{kgN} / \mathrm{ha} / \mathrm{an}$ atteint respectivement 70 et $150 \mathrm{kgN} / \mathrm{ha} / \mathrm{an}$ [35]. L'approche par bilan permet de raisonner globalement les fertilisations azotées capables de maintenir les capacités productives de la prairie. La réduction de la fréquence des retournements des prairies qui en résulte limite les coûts de renouvellement et réduit la pollution des nappes.

\subsection{Bilan de l'azote et risques de pertes en pâturage extensif}

En conditions de pâturage, l'essentiel des pertes se produit par l'intermédiaire des pissats. Dans les pâturages intensifs, les pertes par lessivage et par volatilisation ammoniacale représentent respectivement en moyenne 22 et $16 \%$ de l'azote transitant par les urines de l'herbivore [11]. Dans les pâturages non fertilisés en azote, les pertes sont réduites par une diminution de la surface de pissats résultant de la baisse du chargement [53]. Les recherches devraient vérifier si les pertes d'azote par lessivage sous un pissat sont proportionnelles à l'azote total disponible, somme de la fourniture du sol et de l'ensemble des apports. Dans ce cas, une diminution des apports d'azote minéral et/ou de la fourniture en azote du sol pourraient réduire les risques de pertes sous chaque pissat. Une extensification raisonnée du pâturage doit donc être favorable pour l'environnement.

Tableau I. Dynamique interannuelle de l'azote récolté sur des prairies de montagne dans un régime de fauche sans apports azotés. La dynamique des récoltes à l'apport nul (No) est ajustée à une dynamique exponentielle $\mathrm{No}(\mathrm{t})=\mathrm{No}_{\mathrm{o}} \mathrm{e}^{-\mathrm{kt}} \cdot \log ^{2} / \mathrm{k}$ : durée du régime de fauche nécessaire à une diminution de moitié du No initial. $\mathrm{F}=$ exportation en rythme fauche. $\mathrm{P}=$ exportation en rythme pâture. $\mathrm{D}=$ désherbage chimique des dicotylédones. $\mathrm{PK}=$ compensation des exportations $\mathrm{PK}$ par la fertilisation minérale. Références en sol andique : (1) : données originales ; (2): Arnaud et al. [2].

\begin{tabular}{|c|c|c|c|c|c|c|c|}
\hline Prairic & $\begin{array}{l}\text { Durée } \\
\text { (ans) }\end{array}$ & Rythme & Désherbage & Restitutions & $\begin{array}{c}\text { No initial } \\
\left(\mathrm{kgN} \cdot \mathrm{ha}^{-1}\right)\end{array}$ & $\begin{array}{c}\log ^{2} / \mathrm{k} \\
\text { (ans) }\end{array}$ & $r^{2}$ \\
\hline pelouse & 5 & $\mathrm{~F}$ & - & & 45 & 2.7 & 0.96 \\
\hline \multirow[t]{3}{*}{ (1) } & & $\mathrm{F}$ & $\mathrm{D}$ & & 36 & 3.9 & 0.75 \\
\hline & & $\mathrm{F}$ & - & PK & 32 & 3.9 & 0.67 \\
\hline & & $\mathrm{F}$ & $\mathrm{D}$ & PK & 39 & 4.8 & 0.67 \\
\hline prairic & 5 & $\mathrm{~F}$ & - & & 109 & 6.3 & 0.64 \\
\hline \multirow[t]{3}{*}{ (1) } & & $\mathrm{F}$ & $\mathrm{D}$ & & 118 & 5.6 & 0.64 \\
\hline & & $\mathrm{F}$ & & PK & 122 & 6.8 & 0.49 \\
\hline & & $\mathrm{F}$ & $\mathrm{D}$ & PK & 131 & 5.4 & 0.38 \\
\hline prairie & 7 & $\mathrm{~F}$ & & - & 152 & 5.3 & 0.75 \\
\hline (2) & & $\mathrm{P}$ & - & - & 161 & 4.3 & 0.95 \\
\hline
\end{tabular}


Cependant, il n'est pas certain que la fourniture du sol en azote décroisse après une diminution de l'exploitation ou un abandon de prairies fertiles. Le déclenchement de successions végétales secondaires s'accompagne souvent d'une augmentation de la disponibilité de l'azote [54]. Il en va de même en cas de fauche ou de broyage avec restitution sur place des phytomasses sous forme de mulch [41]. La diminution du chargement sans recours à la fauche exportatrice conduit à des bilans d'éléments voisins de zéro et provoque de faibles changements des caractéristiques des sols [37]. Enfin, les recherches en cours confirment que le niveau de nutrition azotée des espèces n'est pas diminué. Une partie plus importante de l'azote mobilisé dans la croissance des plantes étant recyclé de façon interne, le défaut d'absorption qui en résulte pourrait provoquer au contraire un surcroît d'azote minéral disponible dans le sol. De nouvelles recherches seront nécessaires pour mesurer les effets d'un faible taux de consommation sur le cycle de l'azote et ses conséquences éventuelles pour la fertilité du sol, l'eutrophisation du milieu et le lessivage des nitrates.

\section{CONCLUSION}

Pour une aide à la gestion des systèmes extensifiés, la recherche doit notamment développer une approche écologique fonctionnelle de la dynamique des écosystèmes prairiaux à l'échelle de la parcelle. Trois orientations peuvent être soulignées : i) l'expression des compétitions entre espèces selon leur stratégie de croissance, de morphogenèse et de cycle de vie, pour rendre compte de l'évolution de la structure de la végétation selon les conditions de disponibilité en minéraux et de défoliation ; ii) les conséquences des interactions entre producteurs, consommateurs et décomposeurs, en termes de cycle du carbone et des éléments biogènes, pour rendre compte de la dynamique de fertilité chimique des sols ; iii) la détermination des comportements et des choix alimentaires des troupeaux dans les parcelles hétérogènes. Ces connaissances sur les fonctionnements permettront de tester des modèles de dynamique d'écosystèmes complexes. La mise en relation des fonctionnements écologiques avec les structures de la végétation, et les caractéristiques des sols permettra l'élaboration d'indicateurs des valeurs d'usage et des propriétés dynamiques des prairies, et, par là, des modalités d'interventions techniques qui devront être testées expérimentalement pour être intégrées dans les systèmes de pâturage.

\section{RÉFÉRENCES}

[1] Armstrong R.H., Grant S.A., Common T.G., Beattie M.M., Controlled grazing studies on Nardus grassland : effects of between-tussock sward height and species of grazer on diet selection and intake, Grass Forage Sci. 52 (1997) 219-231.

[2] Arnaud R., de Montard FX., Niqueux M., Essais de fertilisation minérale sur pâturage et sur prairie de fauche en montagne volcanique du Massif Central humide. I. Détermination des doses d'azote et de potasse optimales en conditions de pâturage intensif par des ovins, Fourrages 93 (1983) 3-33.

13] Balent G., Fily M., Dynamique de la végétation selon les pratiques des agriculteurs. Une modélisation à partir des données recueillies dans une vallée des Pyrénées centrales, Études et recherches sur les systèmes agraires et le développement, Inra 23 (1991) 1-48.

[4] Balent G., Magda D., Co-evolution of grassland biodiversity and farm management, Proc. 15th Gen. Meet. EGF (1994) 278-281.

[5] Béranger C., Systèmes extensifs et extensification. Problèmes généraux, in : l'extensification en production fourragère, Fourrages hors série (1992) 13-18

[6] Carrère P., Louault F., Soussana JF, Pichon P., Defoliation of a grass (Lolium perenne L.) - Clover (Trifolium repens L.) mixture continuously grazed by sheep, 5th Int. Rangeland Cong., Salt Lake City, 23-28 July 1995, pp. 80-81.

[7] Carrère P., Schwinning S., Parsons A.J., A spatially explicit simulation of herbivore-sward interactions, 5th BGS Cong. Plymouth, Sept 1997, 2 p. 
[8] Chabosseau J.M., Dedieu B., Extensive livestock farming in Central France : different managements of various projects and situations, Proc. Vth Int. Rangeland Cong., Salt Lake City, USA, 1995, pp. 85-86.

[9] Chabosseau J.M., Dedieu B., La prairie et les interventions mécaniques en système ovin extensif : exemples en Montmorillonnais, Fourrages 151 (1997) 351-372.

[10] Daget P., Poissonet J., Analyse phytologique des prairies. Applications agronomiques, Ann. Agron. 22 (1991) 5-41.

[11] Decau M.L., Delaby L., Roche B., Azopât : une description quantiftée des flux annuels d'azole en prairie pâturée par les vaches laitières. I- Les flux du système sol-plante, Fourrages 151 (1997) 313-330.

112] De Montard F.X., Gachon L., Contribution à l'étude de l'écologie et de la productivité des pâturages d'altitude des Monts Dore. I. Application de l'analyse factorielle des correspondances à l'analyse de la végétation, Ann. Agron. 29 (1978) 277-310.

[13] De Montard F.X., Loiseau P., Gachon L., Nitrogen output in yields and by drainage on natural and sown meadows in the Massif Central, France, EEC Workshop 'Nitrogen fluxes in intensive grassland systems', Wageningen. 12-14 October 1983, 11 p.

[14] Duru M., Dalmières A., Foucras J., Laval L., Le volume d'herbe disponible par animal : un indicateur pour la conduite du pâturage. Application aux vaches allaitantes, Fourrages 150 (1997) 209-223.

[15] Duru M., Balent G., Gibon A., Magda D., Theau J.P., Cruz P., Jouany C., Fonctionnement et dynamique des prairies permanentes. Exemple des Pyrénées centrales, Fourrages 153 (1998) 97-113.

[16] Farrughia A., Decau M.L., Vertès F., Delaby L., En prairie, la balance azotée à l'échelle de la parcelle, Fourrages 151 (1997) 281-296.

[17] Fily M., Balent G., Succession in grazed oldfields: combining the synchronic and diachronic approach through canonical correspondance analysis, J. Veg. Sci. (1992).

[18] Fleury P., Dubouf B., Jeannin B., Forage management in dairy farms: a methodological approach, Agricultural Systems 52 (1996) 199-212.

[19] Fleury P., Le diagnostic agronomique des végétations prairiales el son utilisation dans la gestion des exploitations agricoles. Typologies fondées sur les aptitudes des prairies à remplir des fonctions, thèse, INPL, 1994, 139 p.

[20] Gilibert J., Mathieu A., Une méthode de notation visuelle rapide de l'état des prairies, Fourrages 150 (1997) 191-207.

[21] Gillet F., Gallandat J-D., La phytosociologie synusiale intégrée : objets et concepts, Candollea 46 (1991) 315-340.
[22] Gillet F., Gallandat J.D., Integrated synusial phytosociology: some notes on a new, multiscalar approach to vegetation analysis, J. Veg. Sci. 7 (1996) 13-18.

[23] Grant S.A., Torwell L., Sim E.M., Small J.L., Armstrong R.H., Grazing studies on Nardus grassland: effect of between-tussock sward height and species of grazer on Nardus utilization and floristic composition in two fields in Scottland, J. Appl. Ecol. 33 (1996) 1053-1064.

[24] Grimm V., Schmidt E., Wissel C., On the application of stability concepts in ecology, Ecol. Model. 63 (1992) 143-161.

[25] Grimm V., Wissel C., Babel O.R., the ecological stability discussions: an inventory and analysis of terminology and a guide for avoiding confusion, Oecologia 109 (1997) 323-334.

[26] Guérin G., Bellon S., Analyse des fonctions des surfaces pastorales dans les systèmes de pâturage méditrerrannéens. Recherches sur les systèmes herbagers : quelques propositions françaises, in: Capillon A. (éd.), Études et recherches sur les systèmes agraires et le développement 17 (1990) 147-158

[27] Hoagland B.W., Collins S.L., Gradient models, gradient analysis, and hierarchical structure in plant communities, Oikos 78 (1997) 23-30.

[28] Josien E., Dedieu B., Chassaing C., Étude de l'utilisation du territoire en élevage herbager. L'exemple du réseau extensif bovin limousin, Fourrages 138 (1994) 115-134.

129] L'Homme G., Couhert J.P., Leroy E., Boyer V., Extension et amélioration d'une lande à Callunc, IV $V^{\mathrm{C}}$ Congrès Int. Terres de Parcours, Montpellier, 22-26 avril 1991, 362 p.

[30] Loiseau P., Béchet G., Implications agronomiques de la sélection alimentaire exercée par les ovins sur les constituants d'une végétation pâturée, Ann. Agron. 3 (1975) 289-307.

[31] Loiseau P., Merle G., Influence du mode d'exploitation traditionnel sur l'état des parcours dans la région des Dômes, Fourrages 79 (1979) 37-56.

[32] Loiseau P., Merle G., Production et évolution des landes à callune dans la région des Dômes. Acta Oecol., Oecol. Appl. 2 (4) (1981) 283-298.

133| Loiseau P., Un puissant outils d'amélioration des parcours : le parcage nocturne, Agronomie 3 (1983) 375-385.

[34] Loiseau P., Signification et limite de l'indice de valeur pastorale pour le diagnostic de la valeur agricole des pâturages en moyenne montagne humide, Coll. Phytosociologiques, Phytosociologie et pastoralisme, Paris 14, 1988, pp. 41 1-428.

[35] Loiseau P., El Habchi A., de Montard F.X., Triboï E., Indicateurs pour la gestion de l'azote dans les systèmes de culture incluant la prairie temporaire de fauche, Fourrages 129 (1992) $29-43$. 
[36] Louault F., Carrère P., Soussana J.F., Efficiencies of ryegrass and white clover herbage utilizationnin mixtures continuously grazed by sheep, Grass Forage Sci. 52 (1997) 388-400.

[36b] Magda D., Effects of extensification on invasive population of Chaerophyllum aureum in grassland, J. Veg. Sci. (1998), sous presse.

[37] Marriott C.A., Bolton G.R., Common T.G., Small J.L., Barthram G.T., Effects of extensification of sheep grazing systems on animal production and species composition of the sward, Grassland and Land Use Systems, 16th EGF Meeting, 1996, 505-509.

[38] Morgan C.T., Davies D.A., Fothergill M., Changes associated with the re-introduction of grazing in abandoned upland swards, 5th Res. Conf. BGS, 8-10 Sept. Plymouth, 1997. pp. 45-46.

[38b] Marriot C.A., Carrère P., Structure and dynamics of grazed vegetation, Ann. Zootech. 47 (1998) 359-369.

[38c] Marriot C.A., Hudson G., Hamilton D., Neilson R., Boag B., Handley L.L., Wishart J., Scrimgeour C.M., Robinson D., Spatial variability of total C and D and their stable isotopes in an upland Scottish grassland, Plant Soil 196 (1997) 151-162.

[39] Neubert M.G., Caswell H., Alternatives to resilience for measuring the responses of ecological systems to perturbations, Ecology 78 (1997) $653-665$.

[40] Noy-Meir I., Stability of grazing systems: an application of predator-prey graphs, J. Ecol. 63, (1975) 459-481

[41] Oomes M.J.M., Altena H.J., Decrease in grass land production by management of vegetation and groundwater, Proc. 15th Gen. Meet. EGF, 1997, pp. 328-331.

[42] Orth D., Carrère P., Lefevre A., Duquet P., Michelin Y., Josien E., L'Homme G., L'adjonction de chevaux aux bovins en conditions de sous-chargement modifie-t-elle l'utilisation de la ressource herbagère?, Fourrages 153 (1998) 125-138.

[43] Ouvrage collectif, Aspects biologiques et techniques de la remise en exploitation des hauts pâturages dégradés des Monts Dore, in : Molenat G., Jarrige R. (éd.) Utilisation par les ruminants des pâturages d'altitude et parcours méditerranéens, Inra, 1979, pp. 57-133.

[44] Parsons A.J., Exploiting resource capture-Grassland, in: Monteith J.C., Scott R.K., Unworth M.H. (Ed.), Resource capture by crops, Nottingham Un. Press (1994) 315-349.

[45] Ricou G., Loiseau P., Études sur le recyclage dans l'écosystème prairial. 2- Coprophages et recyclage dans les pelouses montagnardes, Acta Oecol., Oecol. Applicata 5 (1984) 23-41.

[46] Rousseau S., Loiseau P., Structure et cycle de développement des peuplements à Cytisus scoparius L. dans la chaîne des Puys, Acta Oecol. Oecol. Appl. 3 (1982) 155-165.

[47] Simon J.C., Vertès F., Decau M.L., Le Corre L., Les flux d'azote au pâturage. I- Bilans à l'exploitation et lessivage du nitrate sous prairie, Fourrages 151 ( 1997) 249-262.

[48] Simon J.C., Leconte D., Vertes F., Le Meur D., Maîtrise de la pérennité du trèfle blanc dans les associations, Fourrages 152 (1997) 483-498.

[49] Stuefer J.F., Potential and limitations of current concepts regarding the response of clonal plants to environmental heterogeneity, Vegetatio 12 (1996) 55-70

[50] Treweek J.R., Watt T.A., Hambler C., Integration of sheep production and nature conservation: experimental management, J. Environ. Manag. 50 (1997) 193-210.

[51] Van der Maarel E., Vegetation dynamics and dynamic vegetation science, Acta Bot. Neerl., (1996), 421-442.

[52] Van der Maarel E., Pattern and process in the plant community: fifty years after AS Watt, J. Veg. Sci. 7 (1996) 19-28.

[53] Vertès F., Simon J.C., Le Corre L., Decau M.L., Les flux d'azote au pâturage. II- Étude des flux et de leurs effets sur le lessivage, Fourrages 151 (1997) 263-280.

[54] Vitousek P.M., Matson P.A., Van Cleeve K., Nitrogen availability and nitrification during succession: primary, secondary, and old filed seres, Plant Soil 115 (1989) 229-239. 